\title{
Design of FSS Based Radome Wall for Airborne Radar Application
}

\author{
Rashmi A. Pandhare ${ }^{1}$ (), Fateh Lal Lohar ${ }^{2}$ (i), Chandresh Dhote ${ }^{1}$ (i), Yogesh Solunke ${ }^{3}$ \\ ${ }^{I}$ Indian Institute of Information Technology, Nagpur, India \\ rush9ap@gmail.com,dhote.sgsits2017@gmail.com \\ ${ }^{2}$ Second Govt. Engineering College, Jhalawar, India, fatehudml@gmail.com \\ ${ }^{3}$ Indian Institute of Information Technology, Kanchipuram, India, yogsolunke03@gmail.com
}

\begin{abstract}
In this paper, a broad band A-sandwich radome wall structure based on band-pass FSS (frequency selective surface) is proposed for an airborne radar applications. The FSS structure having two square conducting rings, which are embedded on either side of core layer. The conventional and proposed FSS based radome wall is analyzed with different incidence angles $\left(0^{\circ}, 10^{\circ}, \mathbf{2 0}^{\circ}\right.$ and $3^{\circ}$ ) for both TE and TM polarization. The radome wall structure consist of a low density Nomex honeycomb core layer sandwiched between two high density Quartz skin layers. The structure shows the percentage impedance bandwidth of $40.8 \%$ in the frequency range from $7.8 \mathrm{GHz}$ to $11.8 \mathrm{GHz}$ with sharp roll-off characteristics. The proposed novel approach involves high frequency unit cell simulation, which is carried out using EM Simulation Tool. To understand the practical behavior of the proposed structure for radome application the conformal analysis has been also carried out, with respect to different radius. The superior EM performance of the proposed broadband novel radome wall structure makes it suitable for the design of airborne radome.
\end{abstract}

Index Terms - Airborne, FSS, Radome, Radar, TE/TM-Polarization, X-band.

\section{INTRODUCTION}

A radome is a combination of Radar and Dome, it is a cover placed over the antenna that protects the radiating element (Radar Antenna) from its physical environment. Modern radome usually requires electromagnetic compatibility to prevent the antenna from unwanted signals and environmental hazards. Many aircrafts are equipped with high-performance radar which is permeable to a coupling from the nearby transmitting antenna and electromagnetic jam and hence electromagnetic compatibility becomes an important issue nowadays. Another role of the radome is for reduction of antenna Radar Cross Section (RCS) out of the transmitting band [1].

A radome is made up of a material that minimally degrades the electrical performance of the enclosed antenna and effectively transparent to the radio frequency. Today, radomes find a wide range of applications in ground, maritime, vehicular, aircraft, airborne and missile systems [2], [3].

Nowadays a novel airborne radome structure with superior electromagnetic (EM) performance characteristics with an enclosed antenna in the complete operating band is highly demanded. Many techniques were reported for modifying the radome wall configurations to enhance the EM 
Journal of Microwaves, Optoelectronics and Electromagnetic Applications, Vol. 20, No. 4, December 2021 DOI: http://dx.doi.org/10.1590/2179-10742021v20i41341

performance of radome walls. The most basic problem in radome electrical design is the development of a wall configuration that supports the antenna system requirements. The FSSs are electromagnetic structures that periodically arranges conductive patterns or dielectrics having the same shape to reflect or transmit the electromagnetic signal at certain frequencies with the low insertion loss having the same phase current that has been induced by an incident electromagnetic plane wave [4], [5]. The details about the radome wall configuration and FSS are given as below.

\section{A. Characterizing the radome wall configuration}

The radome walls designing is a challenging task in the manufacturing of flight vehicles and the choice of wall construction will depend upon the requirement of applications. The wall must withstand for environmental effects such as erosion, rain, lightning strike, aerodynamic loads, etc. Radomes, on a broad scale, are classified as monolithic and sandwich designs, based on wall configuration as shown in Fig. 1. There are two types of monolithic designs, half-wave radomes (style-a) and electrically thin-walled radomes (style-b). The sandwich designs can be categorized into A-sandwich (style-c), B-sandwich (style-e), C- sandwich and multi-layered dielectric wall (style-d) radomes. One can also introduce an FSS layer into one of the styles to reduce its out-of-band radar cross-section (RCS). The choice of a particular configuration or style depends on the applications.

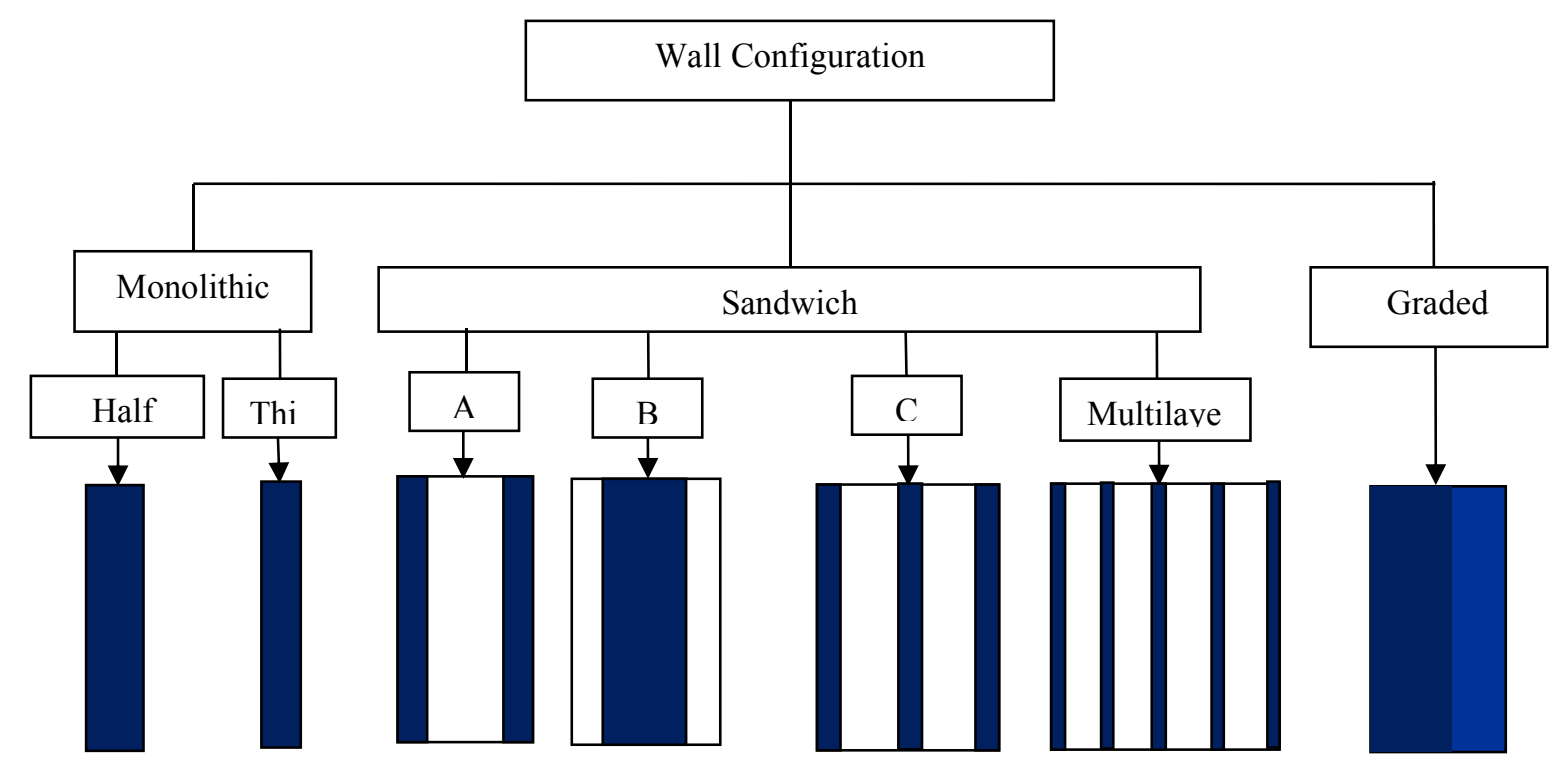

Fig. 1. Radome wall configuration.

The selection of radome material is also equally imperative along with the selection of suitable wall configuration. Electrical, structural, and thermal performances are primary factors in the selection of radome materials. Reliability, length of service, and cost can also be important considerations [2].

\section{B. Radome Wall Material}

To analyze the electrical performance of the radome, it is necessary to estimate the electrical properties of the wall material at various wavelengths. The primary electrical properties of the subject 
Journal of Microwaves, Optoelectronics and Electromagnetic Applications, Vol. 20, No. 4, December 2021

DOI: http://dx.doi.org/10.1590/2179-10742021v20i41341

materials are relative dielectric constant and the loss tangent at the operating frequencies of the radome. The choice of the radome walls material will depend on the applications. There are various types of radome dielectric wall materials present with their electrical and mechanical properties. The dense layer of the wall commonly fabricated using fiber reinforcement material with resin materials. Some of the popular reinforcement materials are S-glass, E-glass, D-glass, Spectra, Quartz, and Kevlar. Where S-glass is high strength glass and E-glass is low cost and has less tensile strength than the S-glass. D-glass is of low strength and high-cost material. However, Spectra consist of polyethylene fibers, which is lightweight, high strength, and low loss material. Quartz is a very low loss but higher in cost than polyethylene, it has the content of silica which is lower in dielectric constant and small loss tangent. Kevlar has higher loss tangent and applicable in high strength application [2], [3], [6].

\section{Frequency Selective Surfaces}

FSSs are planar, periodic structures, which consist of etched elements array, it can be either transmits or reflects EM waves. The FSS conducting strips are modeling by reactive components and it can be represented in other elements such as Jerusalem cross geometry and slots or square loop, it is commonly used in the design of radome, radar absorbing structure, spatial filters, and high impedance surfaces. For transmission behavior, the FSS slot elements are mostly taken in conducting radome wall layer, in order to allow only desired bands of frequency and to eliminate others. Also in many applications, it is desirable to add frequency selectivity to avoid coupling from nearby antennas that may cause the interference [2], [7].

In aerospace applications, FSS is the best choice to use, because at higher incident angles conventional radome performance is poor. The basic design of radome including FSS is band pass FSS array buried in a dielectric material. The FSS array in the solid (monolithic) radome can be inserted into the center of substrate wall. In sandwich structures- FSS commonly immerse in the core layer of substrate. Another approach is to design FSS based radome is thick layers of FSS can be separated by a core layer [3], [7].

\section{Literature Survey}

Many techniques were reported for modifying the radome wall configurations to enhance the EM performance of radome walls .A metamaterial FSS based structure for wideband radome was reported in [8]. The EM performance of modified A sandwich panels by the matching core to that of the skin was studied in [9].The Electromagnetic design and performance analysis of a novel A-sandwich hybrid variable thickness radome (hy-VTR) has been carried out in [10]. The EM performance of the inhomogeneous airborne radomes for circular polarization, where the bandwidth performance and the effect of radome coating and thickness errors were studied in [11]. Design of miniaturized and ultrathin absorptive/transmissive radome with wideband absorbing property has been presented in [12]. An ultra-wide band radome for high-performance and dual-polarized radar and communication Systems 
Journal of Microwaves, Optoelectronics and Electromagnetic Applications, Vol. 20, No. 4, December 2021

DOI: http://dx.doi.org/10.1590/2179-10742021v20i41341

is reported in [13]. An efficient design methodology for sandwich radome panels with a C-band design example was presented in [14].

The conventional radomes suffer from poor EM performance, which requires necessitates the development of new techniques of radome design. Furthermore, rapid progress in the fields of frequency-selective surfaces (FSS) and metamaterial has had a significant impact on the development of the radome technology [15], [16]. Over the last decade, various types of FSS-based radomes were designed to improve the overall EM performance. EM performance analysis of tri-layer metamaterialFSS structure and double-layered thick FSS structure with low-loss dielectric medium between the FSS layers using MM-GSM technique has been reported in [17], [18]. EM design and analysis of frequency selective surface based on substrate-integrated waveguide technology for airborne radome application is proposed in [19]. Analysis of transmission loss and boresight error of a curved fss radome-enclosed antenna is presented in [20]. A Novel design of a frequency selective radome to absorb impinging electromagnetic signals above the operating band of the antenna was presented in [21]. Design of FSS antenna radome system for airborne and ground applications was reported in [22]. Mechanical and electromagnetic behavior of fabricated hybrid composite sandwich radome with a new optimized frequency selective surface proposed in [23]. The various approaches used to have buried FSS structure is monolithic, sandwich, or multilayer radome wall structure to improve the overall EM performance.

In this work, a design and performance analysis of FSS based radome wall for airborne radar application has been carried out. The designing of the radome wall is summarized in the following sections. In section II, the EM design and performance analysis considerations of A-sandwich radome wall have been discussed along with designing of A-sandwich radome wall and embedded of FSS element. In section III, the EM performance analysis of radome wall structure has been carried out for different incidence angles and also for different bending radius using conformal analysis. In section IV, the EM simulation result validation with equivalent ciruit using ADS tool was performed. Finally proposed work has been summarized in the conclusion.

\section{EM DESIGN CONSIDERATION OF A-SANDWICH RADOME WALL}

\section{A. A-Sandwich Radome Wall Structure}

Initially, the A-sandwich radome wall is designed in order to have preliminary information regarding the performance of the conventional radome wall for comparison with the proposed modified version of the radome wall. An A-sandwich radome structure consists of two dielectric skin layers of the higher dielectric constant separated by a core having a lower dielectric constant than the skins. The 3D view and the layered structure of the conventional A-sandwich radome wall are shown in Fig. 2 (a) and (b) respectively. The complete unit cell consists of a total five layers of different dielectric materials. Nomex honeycomb core layer sandwiched in between two high-density Quartz skin layers. 
Journal of Microwaves, Optoelectronics and Electromagnetic Applications, Vol. 20, No. 4, December 2021 DOI: http://dx.doi.org/10.1590/2179-10742021v20i41341

Further, radome wall is coated by a good anti-static and anti-erosion paint to the outer surface of radome wall. Table I show all the properties of the materials selected for a core, skin, and paint layer.

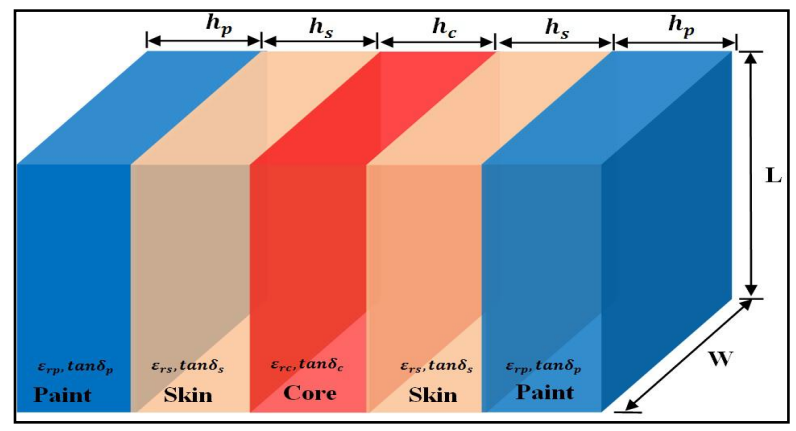

(a)

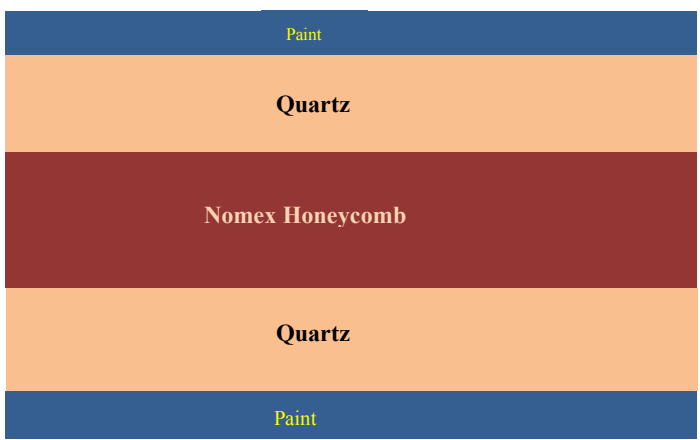

(b)

Fig. 2. (a) 3D view of conventional radome wall; (b) layered structure of conventional radome wall.

TABLE I. DIELECTRIC PROPERTIES OF PROPOSED RADOME WALL

\begin{tabular}{ccccc}
\hline & Material & $\begin{array}{c}\text { Dielectric } \\
\text { Constant }\end{array}$ & Loss Tangent Thickness(mm) \\
\hline Core & Nomex & 1.1 & 0.005 & 1.5 \\
Skin & Quartz & 3.82 & 0.0004 & 2.4 \\
Paint & Radome Paint & 3.32 & 0.0006 & 0.76 \\
\hline
\end{tabular}

Since conventional radomes suffer from poor performance at high incidence angles however FSSbased radome wall gives a flat band pass response and out-of-band sharp roll-off characteristics. Hence, the FSS would be the better option for aerospace applications. Further, in order to improve transmission performance, the conventional A-sandwich radome wall is modified using band-pass double square loop FSS. The two symmetrical double square loop FSS printed on either side of the Nomex honeycomb core layer. The 3D view and the layered structure of the proposed A-sandwich radome wall using a strongly coupled frequency selective surface element is shown in Fig. 3 (a) and (b) respectively. The design parameters of the proposed radome wall with FSS structure are shown in Table II. 


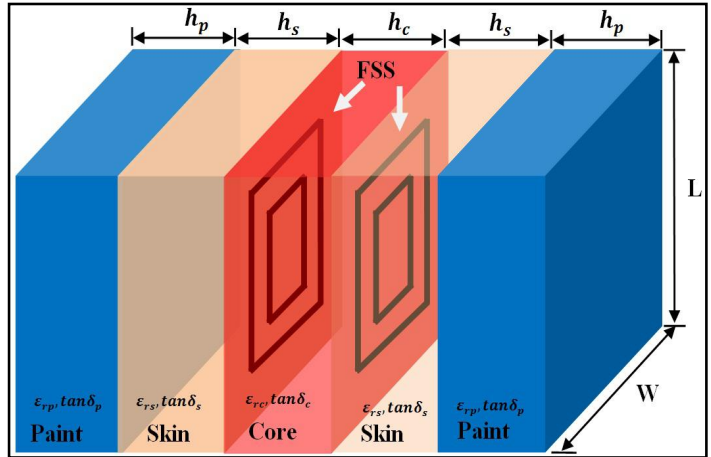

(a)

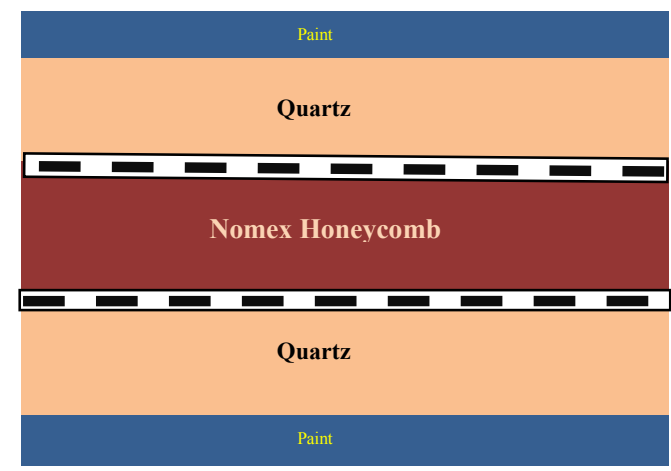

(b)

Fig. 3. (a) 3D view of A-sandwich radome wall with FSS; (b) layered structure of A-sandwich radome wall with FSS.

TABLE II. DESIGN PARAMETERS OF THE PROPOSED RADOME WALL

\begin{tabular}{|c|c|c|c|c|}
\hline Design & Parameters & Value in $\mathrm{mm}$ & Design Parameters & Value in $\mathbf{m m}$ \\
\hline & $\mathbf{L}$ & 15 & $L_{o f}$ & 8.49 \\
\hline & $\mathbf{W}$ & 15 & $L_{i f}$ & 4.24 \\
\hline & $\boldsymbol{h}_{\boldsymbol{p}}$ & 0.76 & $k_{i f}$ & 0.35 \\
\hline & $\boldsymbol{h}_{\boldsymbol{s}}$ & 2.40 & $\boldsymbol{k}_{\boldsymbol{o f}}$ & 0.35 \\
\hline & $\boldsymbol{h}_{\boldsymbol{c}}$ & 1.50 & & \\
\hline
\end{tabular}

\section{B. Design Aspects of Strongly Coupled FSS Element}

The unit cell dimensions of double square loop band-pass FSS and its equivalent circuit model are shown in Fig. 4 (a) and (b) respectively. The band-pass characteristics are achieved by the mutual coupling of two square-loop elements of different dimensions on either side of the core substrate.

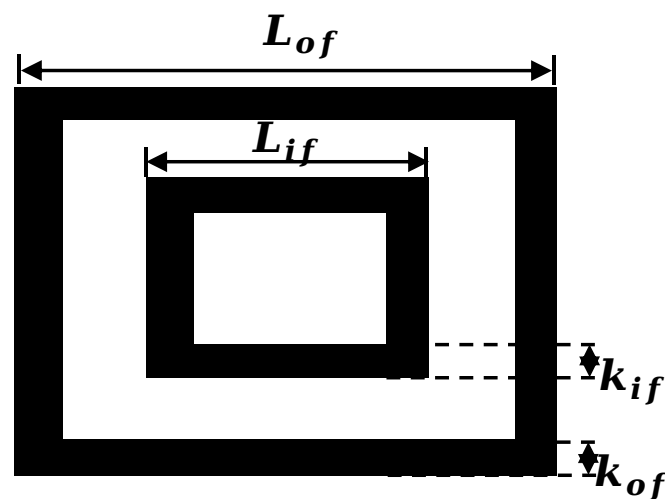

(a)

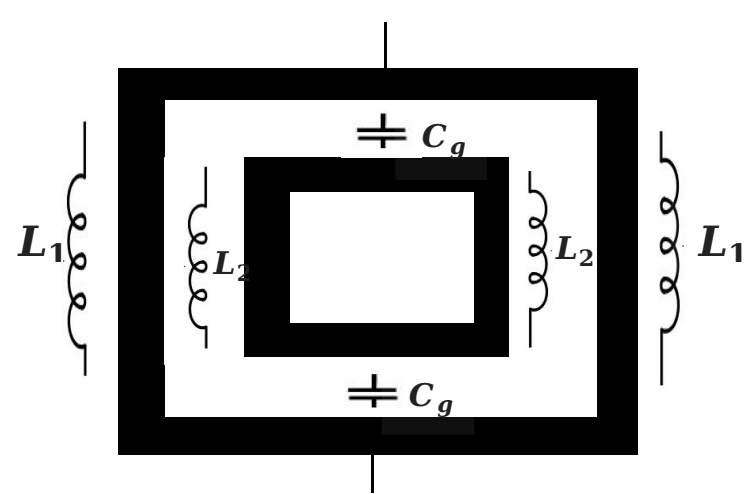

(b)

Fig. 4. (a) Double square loop FSS; (b) Equivalent circuit Model of FSS. 
Journal of Microwaves, Optoelectronics and Electromagnetic Applications, Vol. 20, No. 4, December 2021 DOI: http://dx.doi.org/10.1590/2179-10742021v20i41341

The simplest FSS element is inductive in nature and acting as a pass-band filter. The square loop element is chosen due to its superior performance in terms of stability under various angles of incidence. The length and width of the outer square loop contributes the resonance of lower frequency and inner square loop contributes to the resonance of higher frequency. The combination of both outer and the inner square loop each side of the core substrate behaves as a band pass filter. In Fig. 4 (b) the inductors $\left[L_{1} \mathrm{II} L_{1}\right]$ of outer ring are in parallel combination and similarly inductors $\left[L_{2} \mathrm{II} L_{2}\right]$ for the inner ring. The two-gap capacitors $\left[C_{g}\right]$ are connected in series combination between inner and outer rings. The band-pass characteristics of FSS are achieved by optimizes the parameters of outer and inner square loop for the frequency range of $7.8 \mathrm{GHz}$ to $11.8 \mathrm{GHz}$ with the increment in the bandwidth.

The transmission and reflection coefficients of both conventional radome wall and the proposed radome wall are computed based on Equivalent Transmission Line Method [1]. To estimate this EM performance parameter, the radome wall considered as a multilayered structure with different dielectric layers connected end to end as shown in Fig. 5 (a) and (b). Here $Z_{1}, Z_{2}, Z_{3}$ and $Z_{n}$ represents the impedance of respective layers and $Z_{0}$ is the characteristic impedance of free space. Similarly, the $Z_{p}, Z_{s}$ and $Z_{c}$ are representing the impedances for paint, skin, and core layers respectively as shown in (2). The value of impedance for n-dielectric layer can be computed by $Z_{n}=$ $\sqrt{\frac{\mu_{n}}{\varepsilon_{n}}}[24],[25]$.

\begin{tabular}{|c|c|c|c|c|c|}
\hline & $\begin{array}{l}\text { Layer } \\
1\end{array}$ & Layer 2 & $\begin{array}{l}\text { Layer } \\
3\end{array}$ & & $\begin{array}{l}\text { Layer } \\
\mathrm{n}\end{array}$ \\
\hline $\begin{array}{l}\text { Free } \\
\text { Space }\end{array}$ & & & & י & \\
\hline $\begin{array}{l}Z_{o} \\
=\sqrt{\frac{\mu_{o}}{\varepsilon_{o}}}\end{array}$ & $\begin{array}{l}Z_{1} \\
=\sqrt{\frac{\mu_{1}}{\varepsilon_{1}}}\end{array}$ & $\begin{array}{l}Z_{2} \\
=\sqrt{\frac{\boldsymbol{\mu}_{2}}{\varepsilon_{2}}}\end{array}$ & $\begin{array}{l}Z_{3} \\
=\sqrt{\frac{\mu_{3}}{\varepsilon_{3}}}\end{array}$ & & $\begin{array}{l}Z_{n} \\
=\sqrt{\frac{\mu_{n}}{\varepsilon_{n}}}\end{array}$ \\
\hline
\end{tabular}

(a)

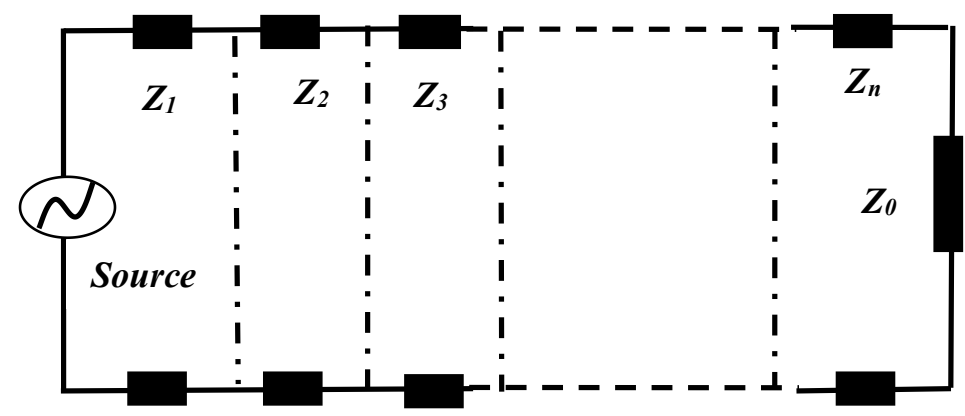

(b)

Fig. 5. (a) Multilayer radome wall structure; (b) Equivalent circuit model of multilayer radome wall structure. 
The Equivalent circuit model of the radome wall with FSS and simplified circuit model of proposed radome wall are shown in Fig. 6 (a) and (b) respectively. Similar equivalent circuit modeling is reported in [25]. The impedance of the proposed FSS radome can be computed based on their equivalent circuit model (ECM), where $A_{f}, B_{f}, C_{f}$ and $D_{f}$ are the $A, B, C, D$ parameters offered by the double square loop FSS elements printed on either side of the core substrate.

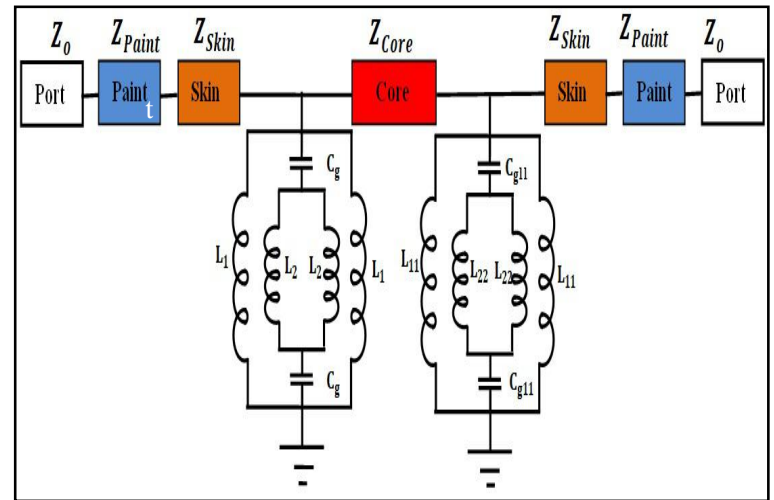

(a)

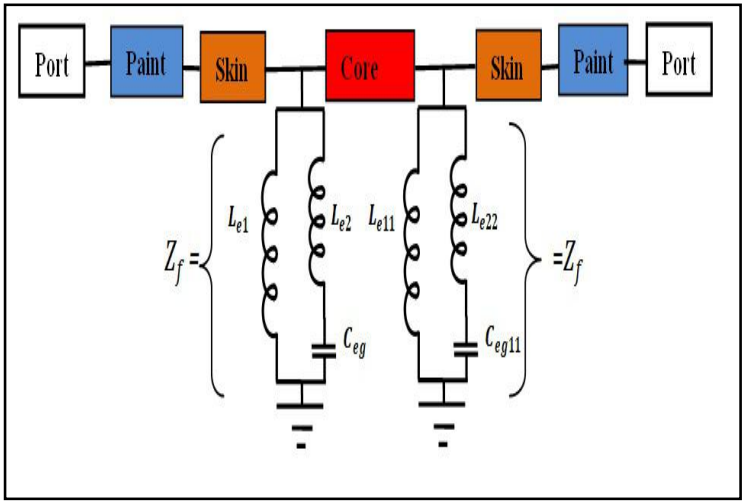

(b)

Fig. 6. (a) Equivalent circuit model of Radome wall with FSS; (b) Simplified circuit of proposed Radome wall.

The $A, B, C, D$ parameters of each layer depend on the material properties of the respective layer, thickness of the material, incidence angles of EM wave on the radome wall and the normalized impedance [25].

The whole unit cell of radome wall with FSS structure can be represented by a single $A B C D$ matrix and it has been obtained by the multiplication of matrices corresponding to individual layers. The voltage-current transmission matrix of the entire radome wall is obtained as follows:

$$
\begin{gathered}
{\left[\begin{array}{ll}
A & B \\
C & D
\end{array}\right]=} \\
{\left[\begin{array}{ll}
A_{p} & B_{p} \\
C_{p} & D_{p}
\end{array}\right]\left[\begin{array}{ll}
A_{s} & B_{s} \\
C_{s} & D_{s}
\end{array}\right]\left[\begin{array}{ll}
A_{f} & B_{f} \\
C_{f} & D_{f}
\end{array}\right]\left[\begin{array}{ll}
A_{c} & B_{c} \\
C_{c} & D_{C}
\end{array}\right]\left[\begin{array}{ll}
A_{f} & B_{f} \\
C_{f} & D_{f}
\end{array}\right]\left[\begin{array}{ll}
A_{s} & B_{s} \\
C_{s} & D_{s}
\end{array}\right]\left[\begin{array}{ll}
A_{p} & B_{p} \\
C_{p} & D_{p}
\end{array}\right]} \\
{\left[\begin{array}{ll}
A & B \\
C & D
\end{array}\right]=} \\
{\left[\begin{array}{cc}
\cos \phi_{p} & j \frac{Z_{p}}{Z_{o}} \sin \phi_{p} \\
j \frac{Z_{o}}{Z_{p}} \sin \phi_{p} & \cos \phi_{p}
\end{array}\right]\left[\begin{array}{cc}
\cos \phi_{s} & j \frac{Z_{s}}{Z_{o}} \sin \phi_{s} \\
j \frac{Z_{o}}{Z_{s}} \sin \phi_{s} & \cos \phi_{s}
\end{array}\right]\left[\begin{array}{ll}
1 & 0 \\
\frac{1}{Z_{f}} & 1
\end{array}\right]\left[\begin{array}{cc}
\cos \phi_{c} & j \frac{Z_{c}}{Z_{o}} \sin \phi_{c} \\
j \frac{Z_{o}}{Z_{c}} \sin \phi_{c} & \cos \phi_{c}
\end{array}\right]} \\
{\left[\begin{array}{ll}
1 & 0 \\
\frac{1}{Z_{f}} & 1
\end{array}\right]\left[\begin{array}{ccc}
\cos \phi_{s} & j \frac{Z_{s}}{Z_{o}} \sin \phi_{s} \\
j \frac{Z_{o}}{Z_{s}} \sin \phi_{s} & \cos \phi_{s}
\end{array}\right]\left[\begin{array}{cc}
\cos \phi_{p} & j \frac{Z_{p}}{Z_{o}} \sin \phi_{p} \\
j \frac{Z_{o}}{Z_{p}} \sin \phi_{p} & \cos \phi_{p}
\end{array}\right]}
\end{gathered}
$$


Here, $\phi$ represents the electrical length of the material layer and $\frac{Z}{Z_{o}}$ is correspond to normalized characteristic impedance of the radome wall layer. Using (3) $\phi$ can be determined by [25].

$$
\phi=\frac{2 \pi h \sqrt{\varepsilon-\sin ^{2} \theta}}{\lambda}
$$

Where, ' $\varepsilon$ ' is a dielectric constant, ' $\theta$ ' is the angle of incidence, ' $h$ ' is the thickness of the radome wall and ' $\lambda$ ' is the free space wavelength. The normalized characteristics impedance for both TE and TM polarization can represent by (4) and (5) respectively. The power transmission and reflection coefficient has been shown in (6) and (7) respectively.

$$
\begin{gathered}
\frac{Z}{Z_{o}}=\frac{\cos \theta}{\sqrt{\varepsilon-\sin ^{2} \theta}} \\
\frac{Z}{Z_{o}}=\frac{\sqrt{\varepsilon-\sin ^{2} \theta}}{\epsilon \cos \theta}
\end{gathered}
$$

The power transmission and reflection coefficient are expressed in (6) and (7) respectively [25].

$$
\begin{aligned}
& P_{t}=\frac{4}{(A+B+C+D)^{2}} \\
& P_{r}=\left(\frac{A+B-C-D}{A+B+C+D}\right)^{2}
\end{aligned}
$$

\section{EM PERFORMANCE ANALYSIS OF THE PROPOSED RADOME WALL}

In this section, the EM performance parameters of the conventional radome wall and the proposed radome wall has been analyzed for different incidence angles, different core thickness and for different bending radius of the wall.The proposed design is simulated using full-wave EM tool with the Finite Element Method (FEM).The simulations are performed according to the topology shown in Fig. 3 and 4, where the unit cell of the structure is placed between Floquet port with periodic boundary conditions to emulate an infinite structure. Initially, the core thickness ' $h c^{\prime}$ ' of the conventional radome wall is optimized as shown in Fig. 7.

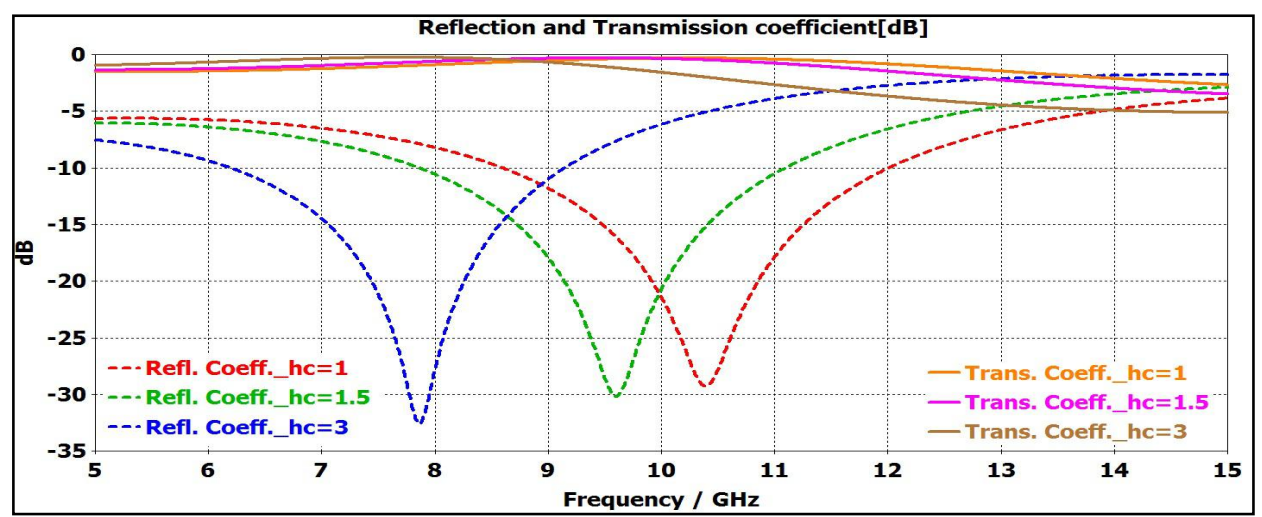

Fig. 7. Optimized core thickness of the conventional radome wall. 


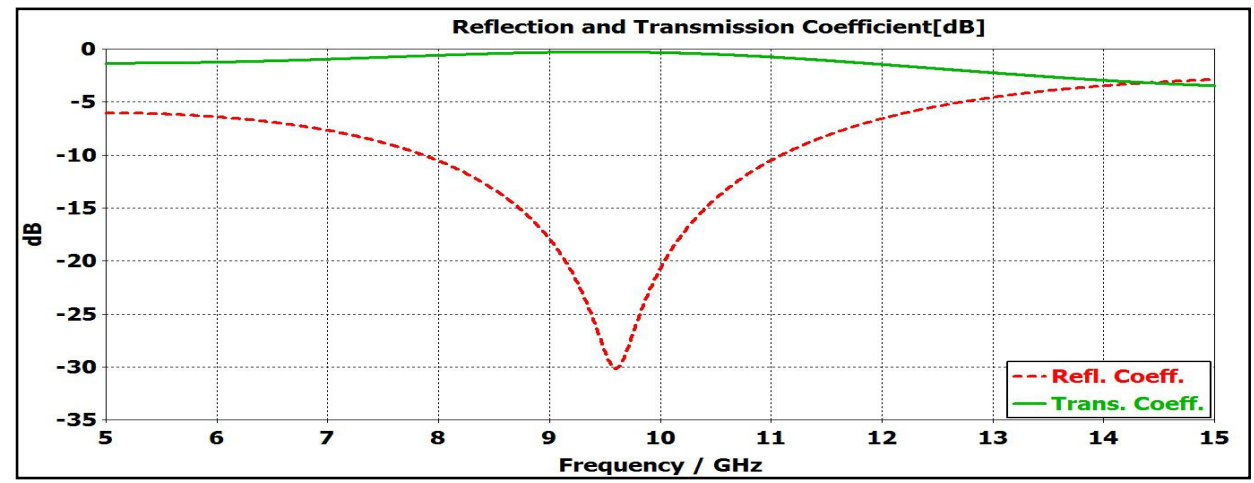

Fig. 8. Conventional Radome wall.

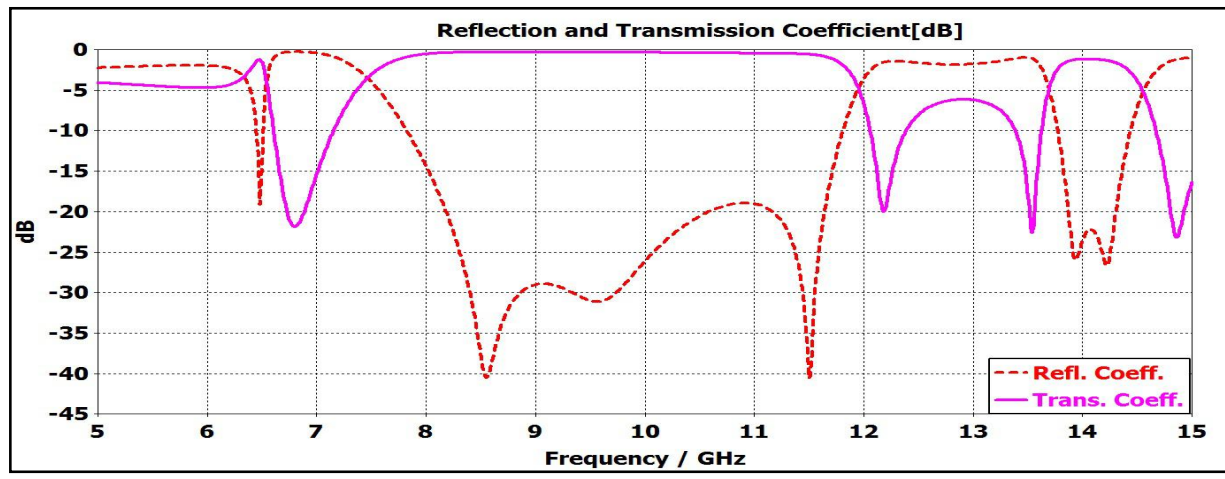

Fig. 9. Proposed radome wall.

It has been observed that, by increasing the value of core thickness $\left(h_{C}=1,1.5\right.$ and $\left.3 \mathrm{~mm}\right)$ of the radome wall, the resonating band is shifting towards the lower frequency band. Therefore, for the $\mathrm{X}$ band application, the $1.5 \mathrm{~mm}$ core thickness has been considered. Fig. 8 and Fig. 9 are showing the reflection and transmission coefficients of the conventional radome wall and the proposed radome wall respectively. As shown in Fig. 8 the frequency response of the conventional radome wall is resonating at $9.5 \mathrm{GHz}$ centre frequency with $3 \mathrm{GHz}(8 \mathrm{GHz}$ to $11 \mathrm{GHz})$ bandwidth. However, it can be observed from Fig. 9 that there is an enhancement of bandwidth from $3 \mathrm{GHz}$ ( $8 \mathrm{GHz}$ to $11 \mathrm{GHz}$ ) to $4 \mathrm{GHz}(7.8 \mathrm{GHz}$ to $11.8 \mathrm{GHz})$ due to the integration of FSS in the conventional radome wall.

\section{A. Incident Angle}

For the applications of airborne radome, the incidence angles for plane waves should have to consider carefully because large incidence angles will make significant effects on the EM performance. The EM performance of the proposed radome wall is better than the conventional radome wall due to higher bandwidth and sharp roll-off characteristics. The proposed structure has been studied for different incident angles $\left(0^{\circ}, 10^{\circ}, 20^{\circ}\right.$ and $\left.30^{\circ}\right)$ and the simulated transmission and reflection coefficient for the both TE and TM polarization shown in Fig.10 and Fig. 11 respectively. It has been observed that for small incidence angles (less than $10^{\circ}$ ), the pass-bands characteristics for radome wall do not 
Journal of Microwaves, Optoelectronics and Electromagnetic Applications, Vol. 20, No. 4, December 2021 DOI: http://dx.doi.org/10.1590/2179-10742021v20i41341

change significantly but as increases the angle of incident from $10^{\circ}$ to $30^{\circ}$, the bandwidth becomes narrower.

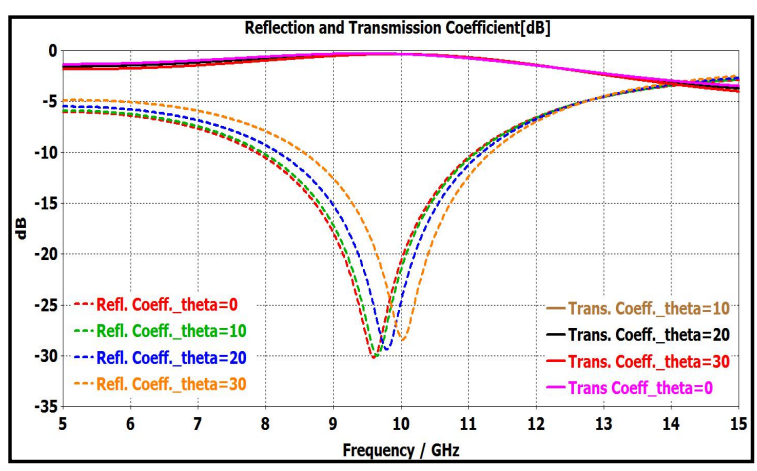

(a)

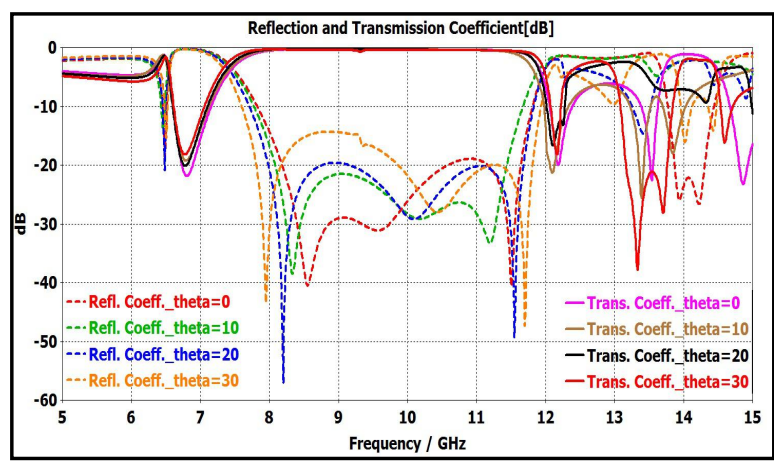

(b)

Fig. 10. TE-Polarization Reflection and Transmission Coefficient with different Incident angles (a) Conventional radome wall; (b) Proposed radome wall.

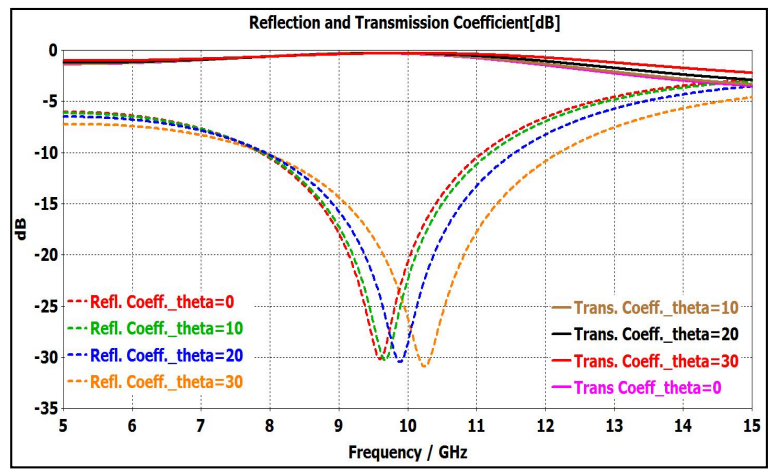

(a)

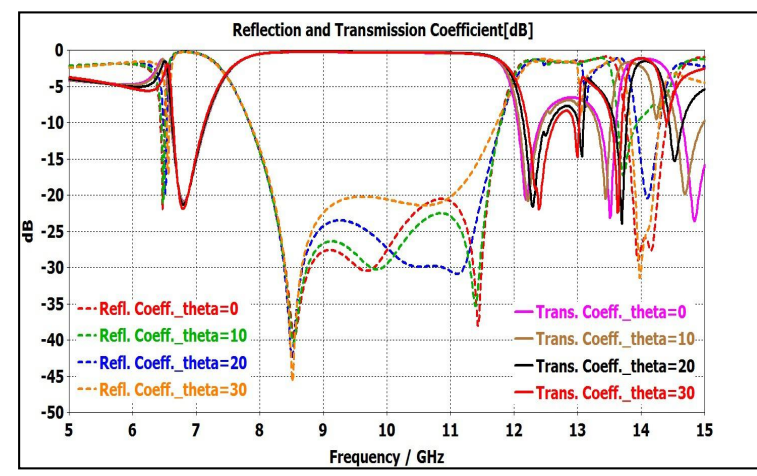

(b)

Fig. 11. TM-Polarization Reflection and Transmission Coefficient with with different Incident angles (a) Conventional radome wall; (b) Proposed radome wall.

\section{B. Conformal Analysis}

Fig. 12 show the conformal analysis of unit cell for the proposed radome wall structure, whereas Fig. 13 and Fig. 14 are representing the almost stable transmission and reflection EM performance in the frequency range of $7.8 \mathrm{GHz}$ to $11.8 \mathrm{GHz}$ at various bending radius $(\mathrm{R}=90,180,270$ and $360 \mathrm{~mm})$ for both TE and TM polarization. It has been observed that there is a minor deviation in EM performance by increasing the bending radius (up to $360 \mathrm{~mm}$ ) of the proposed radome wall. Hence, this is a desirable condition for the airborne radome.

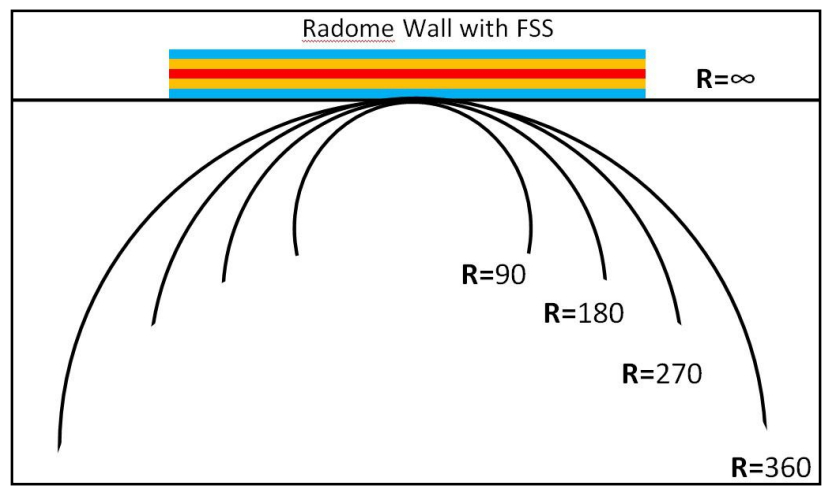

Fig. 12. Conformal analysis of proposed radome wall.

Brazilian Microwave and Optoelectronics Society-SBMO Brazilian Society of Electromagnetism-SBMag received 9 May 2021; for review 22 May 2021; accepted 18 Aug 2021 (C) 2021 SBMO/SBMag (cc) BY 


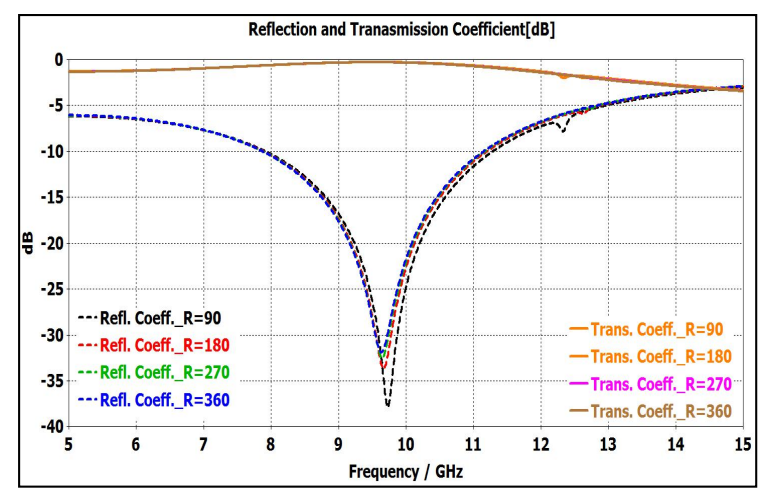

(a)

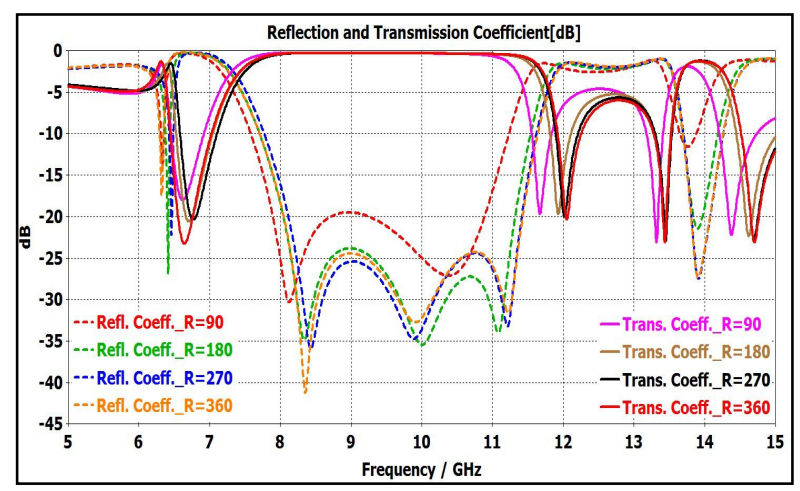

(b)

Fig. 13. TE-Polarization Reflection and Transmission Coefficient with different bending radius (a) Conventional radome wall (b) Proposed radome wall.

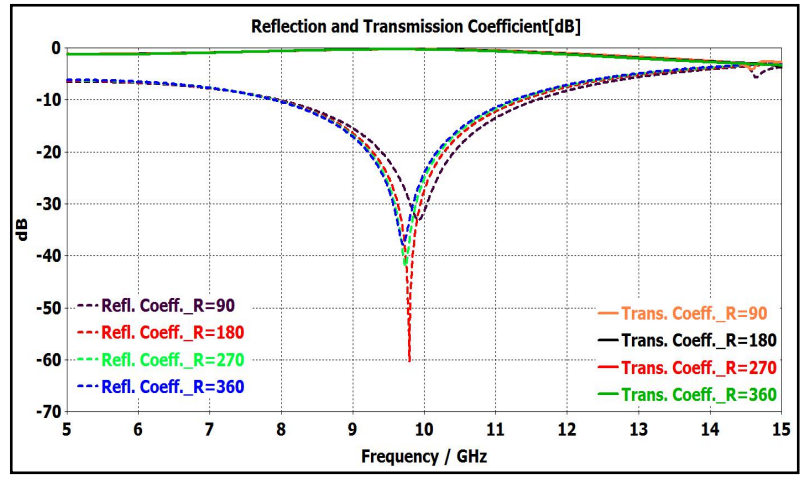

(a)

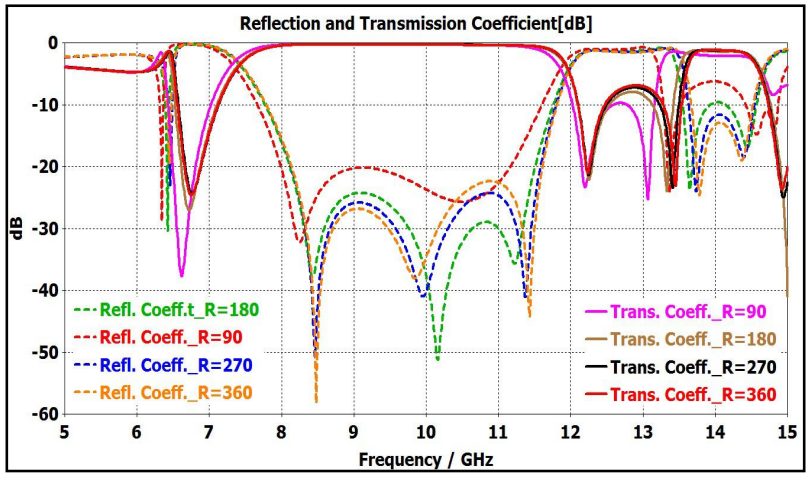

(b)

Fig. 14. TM-Polarization Reflection and Transmission Coefficient different bending radius (a) Conventional radome wall; (b) Proposed radome wall.

\section{EM SIMULATION RESULTS VALIDATION WITH EQUIVALENT CIRCUIT}

The equivalent circuit simulation is illustrated in Fig. 15(a). The ADS software is used to optimise the RLC parameters of the circuit.The transmission and reflection coefficint of equivalent circuits are aproximately similar to the EM simulated results after optimization of circuit parameters, as shown in Fig. 15(b). 


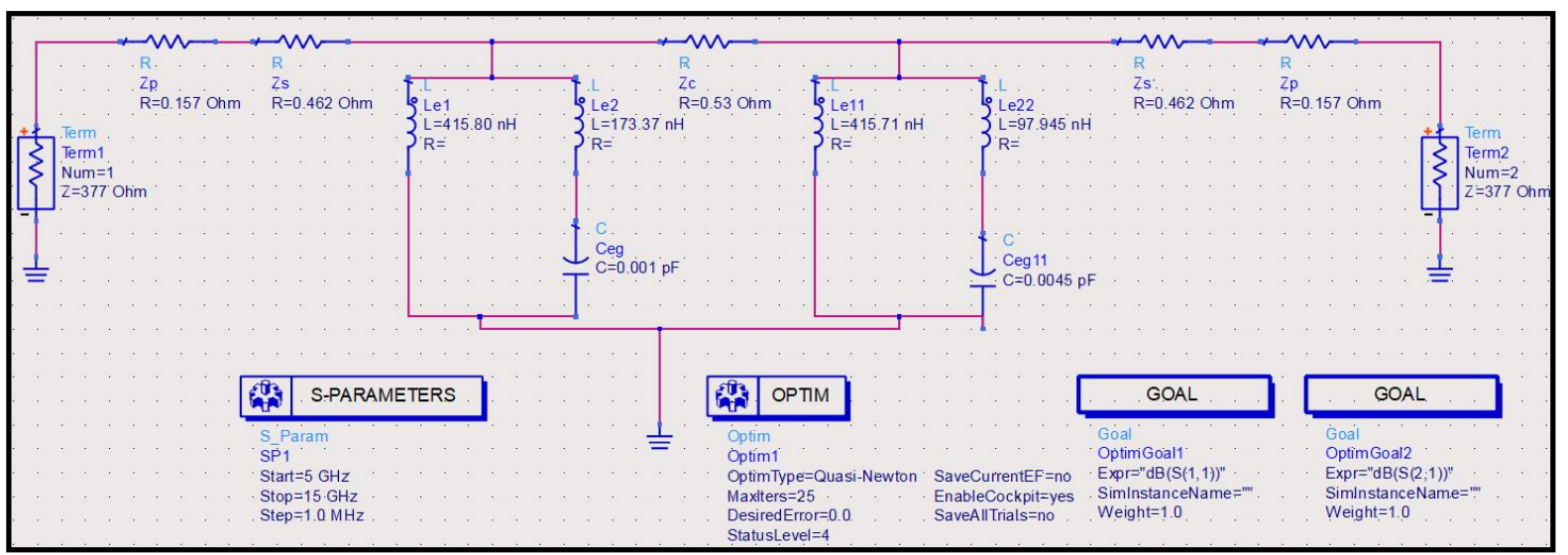

(a)

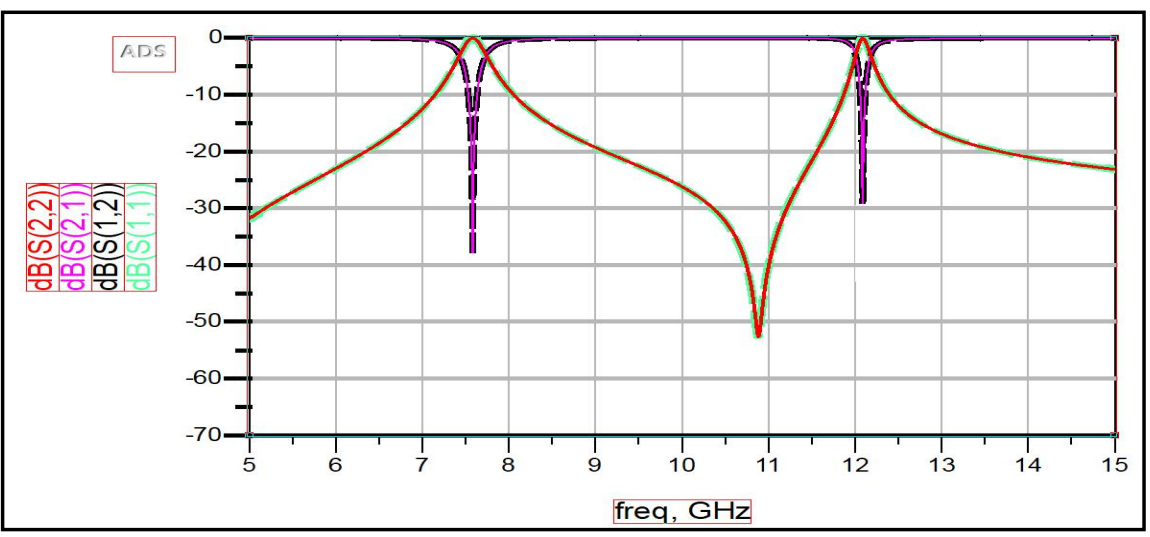

(b)

Fig. 15. (a) Equivalent circuit simulation using ADS tool, (b) Reflection and transmission coefficient of Circuit.

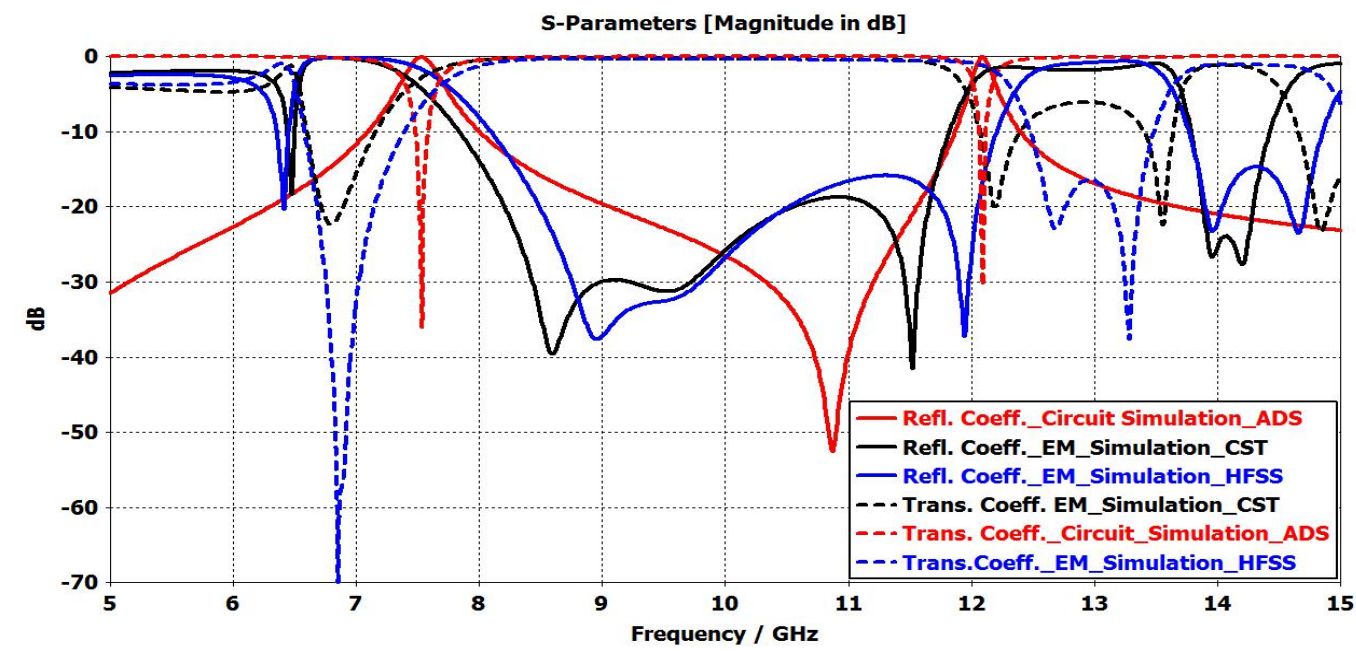

Fig. 16. Proposed radome wall results validation with HFSS, CST and ADS Circuit Simulation.

As a result, the proposed radome wall equivalent circuits validate the analytical to simulated characteristic's relationship. In addition, as shown in the Fig. 16, the CST EM tool simulated characteristics are compared to the HFSS EM tool, as well as with circuit simulation results. In this case, all simulation results from various tools are approximately similar.

Brazilian Microwave and Optoelectronics Society-SBMO received 9 May 2021; for review 22 May 2021; accepted 18 Aug 2021 Brazilian Society of Electromagnetism-SBMag 
Journal of Microwaves, Optoelectronics and Electromagnetic Applications, Vol. 20, No. 4, December 2021 DOI: http://dx.doi.org/10.1590/2179-10742021v20i41341

\section{CONCLUSION}

The conventional radome wall embedded with FSS has been demonstrated in this paper. The EM performance of the conventional radome wall has been compared with proposed radome wall at different incident angles for both TE and TM polarization. The conformal analysis of the unit cell also has been carried out for different bending radius of the radome wall. The radome wall structure with FSS exhibits very good band-pass characteristics and sharp roll-off with enchantment in the bandwidth of $4 \mathrm{GHz}(7.8 \mathrm{GHz}$ to $11.8 \mathrm{GHz})$. The EM Simulation results validation with Equivalent circuit has been carried out and the CST EM tool simulated characteristics are compared to the HFSS EM tool, as well as with circuit simulation results and found the good agreement in all simulated results.Further the superior EM performance of the proposed radomewall structure makes it suitable for the design of airborne radomes. The future work is to analysis of thermal and mechanical properties of proposed radome wall.

\section{REFERENCES}

[1] X. Yuan, X. Yuan, “A transmissive/absorbing radome wit double absorbing band," Microwave and Optical Technology Letters., vol. 58, no. 8, pp. 2016-2019, August 2016.

[2] D. J. Kozako, Analysis of radome-enclosed antennas. Artech House, 2010.

[3] A. Parameswaran, H.S. Sonalikar, "Design of airborne radome using novel temperature dependent electromagnetic modeling," Progress In Electromagnetics Research C, vol. 104, pp. 37-52, 2020.

[4] Q. Chen, Y. Fu, "A planar stealthy antenna radome using absorptive frequency selective surface," Microwave and Optical Technology Letters, vol. 56, no. 8, pp. 1788-1792, August 2014.

[5] I. G. Lee, Y. J. Kim, I. P. Hong, "Practical absorptive frequency selective surface based on printed electronics technology for radome applications," Microwave and Optical Technology Letters, vol. 61, no. 12, pp. 2709-2719, March 2019, DOI: 10.1002/mop.31959.

[6] G. Gampala, M. Vogel, C. E Reddy, "Efficient design and analysis of airborne radomes," Microwave Journal, vol. 58, pp. 28-36, May 2015.

[7] K. K. Varikuntla, R. Singaravelu, "Design of a hybrid A-sandwich radome using a strongly coupled frequency selective surface element," International Journal of Microwave and Wireless Technologies, vol. 12, no. 8, pp. 738-748, October 2020.

[8] S. Narayan, R. M. Jha, “A novel metamaterial FSS-based structure for wideband radome applications," CMC-Computers, Materials \&Continua, vol. 31, no. 2, pp. 97-108, 2013.

[9] V. Chase, "Investigation of improved composites for radome applications," Symposium on Electromagnetic Windows, 13th, Atlanta, pp. 21-23, September 1976.

[10] R.U. Nair and R.M. Jha, "Novel A-sandwich radome design for airborne applications," Electronics Letters, vol. 43, no. 15, pp. 787-788, July 2007.

[11] Xu, W., P. Li, and Y. Qiu, "Electromagnetic performance analysis of inhomogeneous airborne radomes for circular polarization applications," IEEE Antennas and Wireless Propagation Letters, vol. 18, pp. 74-78, 2019.

[12] B. Yi, P. Liu, G. Li, Y. Dong, "Design of miniaturized and ultrathin absorptive/transmissive radome with wideband absorbing property," Microwave and Optical Technology Letters, vol. 58, no. 8, pp. 1870-1875, August 2016.

[13] Z. Qamar, J. L. Salazar-Cerreno, N. Aboserwal, "An Ultra-Wide Band Radome for High-Performance and DualPolarized Radar and Communication Systems," in IEEE Access, vol. 8, pp. 199369-199381, Oct 2020, doi: 10.1109/ACCESS.2020.3032881.

[14] H. Aliakbarian, M. Taherkhani, M. Mokhtari, F. Nazari, \& O. Shekoofa, “An Efficient Design Methodology for Sandwich Radome Panels: A C-band Design Example", IET Science, Measurement \& Technology, vol. 14, no. 7, pp. 808-816, 2020.

[15] S. Narayan, R.M. Jha, "Electromagnetic techniques and design strategies for FSS structure applications [antenna applications corner]," IEEE Antennas and Propagation Magazine, vol. 57, no. 5, pp. 135-158, Ovtober 2015.

[16] B.A. Munk, Element types: a comparison. In: Frequency Selective Surfaces: Theory and Design, Wiley; p. 50-51, 2000.

[17] S. Narayan, K. Arun, R. Jha, "EM analysis of tri-layer metamaterial FSS for radome applications," In: International Conference on Computational \& Experimental Engineering and Sciences ICCES'12, 2012. 
Journal of Microwaves, Optoelectronics and Electromagnetic Applications, Vol. 20, No. 4, December 2021 DOI: http://dx.doi.org/10.1590/2179-10742021v20i41341

[18] S. Narayan, K. Prasad, R. U. Nair, and R. M. Jha, "A novel EM analysis of double-layered thick FSS based on MMGSM technique for radome applications," Progress In Electromagnetics Research, vol. 28, pp. 53-62, 2012.

[19] V. K. Kanth, S. Raghavan, "EM Design and Analysis of Frequency Selective Surface Based on Substrate-Integrated Waveguide Technology for Airborne Radome Application", IEEE Transactions On Microwave Theory And Techniques, vol. 67, no. 5, pp. 1727 - 1739, May 2019.

[20] H. Shin, D. Yoon, D. Y. Na and Y. B. Park , "Analysis of Transmission Loss and Boresight Error of a Curved FSS Radome-Enclosed Antenna,” IEEE Access, vol. 9, pp.95843-95852, July 2021, DOI. 10.1109/ACCESS.2021.3094526.

[21] F. Costa, A . Monorchio, "A frequency selective radome with wideband absorbing properties," IEEE Transactions on Antennas and Propagation, vol. 60, no. 6, pp. 2740-2747, June 2012.

[22] H. U. Tahseen, L. Yang, X. Zhou, "Design of FSS-antenna-radome system for airborne and ground applications," IET Communications, April 2021, https://doi.org/10.1049/cmu2.12181.

[23] H. Rafieipour, A. Setoodeh, A. K.-T. Lau, "Mechanical and electromagnetic behavior of fabricated hybrid composite sandwich radome with a new optimized frequency selective surface", Composite Structures, vol. 273, pp. 114256, ISSN 0263-8223,Oct 2021, https://doi.org/10.1016/j.compstruct.2021.114256.

[24] M. N. Sadiku, "Principles of Electromagnetics," Oxford University Press Inc. First India edition, 2009. 2. Bharathidasan Eng. College, 2015.

[25] R. Nair, S. Vandana, S. Sandhya, R. M. Jha, "Temperature-dependent electromagnetic performance predictions of a hypersonic streamlined radome,” Progress In Electromagnetics Research, vol.154, pp.65-78, 2015.

[26] F. A. Wang, "Tri-band Angularly Stable Frequency Selective Surface with Controllable Resonances for EM Shielding,"Frequenz, vol.74, no.1-2, pp. 25-31, 2020.

[27] A. P. Aparna, H. S. Sonalikar, "Temperature dependent electromagnetic design of dielectric wall for airborne applications," IEEE Indian Conference on Antennas and Propagation (InCAP), 2019. 